\title{
Adequacy of reporting monitoring regimens of risk factors for cardiovascular disease in clinical guidelines: systematic review
}

\author{
Ivan Moschetti, visiting research fellow, ${ }^{1}$ Daniel Brandt, medical student, ${ }^{2}$ Rafael Perera, university lecturer in \\ statistics, ${ }^{1} \mathrm{M}$ Clarke, director, UK Cochrane Centre, ${ }^{3}$ Carl Heneghan, clinical reader in evidence based \\ medicine $^{1}$
}

\begin{tabular}{l}
${ }^{1}$ Department of Primary Health \\
Care, University of Oxford, Oxford, \\
UK \\
${ }^{2}$ Department of Medicine, \\
University of Toronto, Toronto, \\
Canada \\
${ }^{3}$ UK Cochrane Centre, National \\
Institute of Health Research, \\
Oxford, UK \\
\hline Correspondence to: C Heneghan
\end{tabular} carl.heneghan@dphpc.ox.ac.uk

Cite this as: BMJ 2011;342:d1289 doi:10.1136/bmj.d1289

\section{ABSTRACT \\ Objective To assess the reporting of monitoring recommendations in guidelines on the prevention and treatment of cardiovascular disease. \\ Data sources Medline, Trip database, National Guideline Clearinghouse, and databases containing guidelines published from January 2002 to February 2010.}

Data selection Three major risk factors for cardiovascular disease: cholesterol level, smoking, and hypertension. The primary outcome was the frequency with which the guidelines dealt with monitoring of risk factors.

Secondary outcomes were completeness of monitoring recommendations, defined by the presence of what to monitor, when to monitor, what to do if the targets or variables were not met, and the reported level or strength of the evidence.

Results 117 guidelines were identified, 84 (72\%) of which contained a section on lipids. Of those guidelines with a section on lipids, $53 \%(n=44)$ provided no information or specific recommendations on what to monitor, $51 \%$ $(n=43)$ provided no information on when to monitor, and $64 \%(n=54)$ provided no guidance on what to do if the target was out of range. Guidelines for hypertension $(n=79)$ and smoking $(n=65)$ were little better, with $63 \%$ $(n=50)$ and $54 \%(n=35)$, respectively, providing no recommendation for what to monitor. The number of guidelines that explicitly referenced the level of evidence for monitoring was low, with most of the recommendations based on weak levels of evidence.

Conclusion Many guidelines for cardiovascular disease do not report clearly what to monitor and what to do if a change is detected. If no evidence is available to support a specific monitoring schedule, this should be explicit in the guideline, with a description of the new research that would fill the gap.

\section{INTRODUCTION}

One of the most common consultations in healthcare is for the management of cardiovascular disease and subsequent monitoring. Information derived from monitoring drives changes in therapy, clinical workload, and sometimes prognosis. Although initiatives to improve the quality of reporting in healthcare research focus on validity, presentation of results, and treatment decisions, ${ }^{1}$ less attention has been given to the description and accuracy of the monitoring processes that underpin routine clinical practice for the risk management of cardiovascular disease.

Apart from age and sex, three modifiable risk factors - smoking, blood pressure, and cholesterol levelmake a substantial contribution to the risk of cardiovascular disease, with high levels accounting for $80 \%$ of all cases of premature coronary heart disease in high income countries. ${ }^{23}$ Although guidelines often report the timing interval for screening people at low risk of coronary disease, ${ }^{4}$ in our clinical practice we have difficulty delineating the actions required during followup of those at increased risk. For example, most lipid management guidelines clearly state the number and interpretation of initial measurements but are less specific (and in some cases discordant) about the subsequent monitoring decisions and actions to be taken in clinical practice. $^{5}$

A better understanding of monitoring processes for cardiovascular disease could impact substantially on clinical decisions and overall costs to the healthcare system. For example, lipid panels were the third highest contributors to the growth in tests by Medicare between 2000 and $2004 .{ }^{6}$ Clinical guidelines aim to raise the overall quality of care by standardising the decision making process; however, no systematic examination has been done of the reporting of monitoring recommendations within such guidelines. Yet they form a substantial driver of workload in healthcare. To understand if this basic need for knowledge is being met by current guidelines, we undertook a systematic review of the reporting of monitoring strategies in cardiovascular disease prevention guidelines for three major risk factors - cholesterol level, smoking, and hypertension-determining whether the guidelines reported what to monitor, how frequently to 
monitor, and what to do if the variable index was out of range.

\section{METHODS}

We included published guidelines tackling the prevention or treatment of three risk factors for cardiovascular disease: dyslipidaemia, hypertension, and tobacco consumption. We also considered guidelines including the three risk factors developed for the following specific disease areas: diabetes, chronic kidney disease, hypertension, dyslipidaemia, smoking, general practice, surgery, obesity, infertility, screening in adults, and HIV. We restricted the search to English language guidelines published or updated between January 2002 and February 2010. Where more than one version of the same guideline was found we included only the up to date version. We excluded guidelines on cardiovascular disease in pregnant or paediatric populations.

\section{Information source}

We searched Medline using the strategy ("Cardiovascular Diseases/prevention and control" OR "Cardiovascular Diseases/therapy") AND ("Hypertension" OR "Dyslipidemias” OR "Tobacco Use Cessation") limiting to Practice Guideline [publication type] and English language articles. We supplemented this by searching the Trip database and the databases of the National Guideline Clearinghouse. We also searched several databases or websites that produced more than one guideline (see web extra appendix 1 for the full search strategy). Two reviewers (DB and IM) independently assessed all abstracts for potential eligibility, with discrepancies resolved by consensus.

\section{Types of intervention and outcomes}

For the purposes of this review we defined monitoring as the periodic measurement of a chronic or recurrent condition. ${ }^{7}$ We considered two forms of monitoring: before treatment, as part of recommendations regarding screening, because treatment should not start until sufficient measurements for a firm baseline have been obtained, and during treatment, because once the target is achieved the objective of monitoring is to ensure that risk factors stay within reasonable limits.

The primary outcome was to identify the extent to which monitoring was tackled within the guidelines. The secondary outcome was the completeness of monitoring recommendations, defined by the presence of three components: a specific target or variable to monitor, the frequency with which the specific target should be monitored, and changes to consider if the monitored targets or variables were not met. In guidelines that reported specific time intervals for monitoring we also report the specific statement and the level or presence of evidence underpinning the recommendations (see web extra appendix 2).

\section{Data collection and analysis}

Two reviewers (DB and IM) independently extracted data from included guidelines on the year of publication, country or region, principal disease, whether the guideline dealt with primary or secondary prevention, the length of the guideline, the presence of a specific section dealing with risk factors, and information about monitoring (see web extra appendix 3). Information was also extracted on whether there was a definitive target or variable to monitor, when or how often it should be monitored, and what to do if the recommended target had not been met (see web extra appendix 4 for the definition used to characterise the guidelines). The inter-rater reliability (joint probability of agreement - that is, number of times each rating was assigned by each rater, divided by the total number of ratings) was 0.81 . Any discrepancies were resolved by consensus and a common, single dataset was established.

After the initial analysis and results were available, two authors (DB and $\mathrm{CH}$ ) independently identified guidelines produced by a national or international initiative, or developed by a guideline organisation or programme with high output. We then carried out a retrospective subgroup analysis of these guidelines to test whether our results differed when looking only at what might be expected to be "higher quality" guidelines.

\section{RESULTS}

Overall, 874 abstracts were screened, including 122 guidelines published or updated between 2002 and February 2010 on the prevention and treatment of cardiovascular disease. Five were excluded because the full text could not be accessed. Data were extracted from the remaining 117 guidelines, of which 90 (77\%) were either a product of a national or international initiative or developed by a guideline organisation with high output (see web extra appendix 5 for the full reference list of guidelines). All results presented were broadly similar between this subset of guidelines and those produced outside of a national or international initiative or a guideline organisation.

In total, 84 of $117(72 \%)$ guidelines contained a section on lipids, and of these $63 \%(\mathrm{n}=53)$ mentioned monitoring (table). Fifty three per cent $(n=44)$ provided no information or no specific recommendations (that is, those that would be useful in clinical practice) on what to monitor, $51 \%(\mathrm{n}=43)$ provided no information on when to monitor, and $64 \%(\mathrm{n}=54)$ provided no guidance on what to do if the target was out of range (table).

In guidelines that report on when to monitor for lipids, the information reported differed greatly. For example, some guidelines were vague: "lipids should be checked regularly," or "at regular intervals," whereas others compounded the confusion: "risk factors assessment should be undertaken somewhere between one to three years,"10 "every three to five years in all patients," 11 "every five years," or "every one or two years after middle age."12 In addition, guidelines that reported individualised advice for different categories of risk were rare. ${ }^{12} 13$

Seventy nine of $117(68 \%)$ guidelines contained a section on hypertension, and about half of these 
Proportion of guidelines dealing with management of risk factors for cardiovascular disease, and completeness of monitoring recommendation

\begin{tabular}{|c|c|c|c|c|c|c|c|c|c|c|c|c|}
\hline \multirow[b]{3}{*}{ Risk factor } & \multirow{3}{*}{$\begin{array}{l}\text { No }(\%) \text { with } \\
\text { section on } \\
\text { risk factor }\end{array}$} & \multirow{3}{*}{$\begin{array}{c}\text { No (\%) } \\
\text { including } \\
\text { monitoring }\end{array}$} & \multirow{3}{*}{$\begin{array}{l}\text { No (\%) with } \\
\text { section on } \\
\text { management }\end{array}$} & \multicolumn{9}{|c|}{ Completeness of monitoring recommendation } \\
\hline & & & & \multicolumn{3}{|c|}{ What to monitor } & \multicolumn{3}{|c|}{ When to monitor } & \multicolumn{3}{|c|}{$\begin{array}{l}\text { What to do if target } \\
\text { is out of range }\end{array}$} \\
\hline & & & & $\begin{array}{l}\text { Not } \\
\text { reported }\end{array}$ & $\begin{array}{c}\text { Non- } \\
\text { specific* }\end{array}$ & Specific & $\begin{array}{l}\text { Not } \\
\text { reported }\end{array}$ & $\begin{array}{c}\text { Non- } \\
\text { specific* }\end{array}$ & Specific & $\begin{array}{l}\text { Not } \\
\text { reported }\end{array}$ & $\begin{array}{c}\text { Non- } \\
\text { specific* }\end{array}$ & Specific \\
\hline Lipids & $84(72)$ & $53(63)$ & $34(40)$ & $31 \dagger$ & 13 & 40 & $31 \dagger$ & 12 & 41 & 31 & 23 & 30 \\
\hline Hypertension & $79(68)$ & $40(51)$ & $26(32)$ & 39 & 11 & 29 & 39 & 12 & 28 & 39 & 16 & 24 \\
\hline Smoking & $65(55)$ & $37(57)$ & $19(29)$ & 35 & - & 30 & 28 & 17 & 20 & 28 & 14 & 23 \\
\hline
\end{tabular}

*Not useful in practice.

†4 guidelines provided no information or non-specific information.

$(\mathrm{n}=40)$ mentioned monitoring (table). Sixty three per cent $(\mathrm{n}=50)$ provided no information or no specific recommendations for what to monitor, 64\% $(n=51)$ for when to monitor, and $69 \%(\mathrm{n}=55)$ for what to do if the target was out of range (table).

The monitoring of hypertension is an example of the confusion caused by discordant information in different guidelines. The duration for monitoring varied, with clinicians being able to choose among: every three years, ${ }^{14}$ at least every two years, ${ }^{15}$ at least annually, ${ }^{16}$ every visit and at least every six months, ${ }^{17}$ each healthcare encounter, ${ }^{18}$ and as needed, ${ }^{18}$ or simply "monitor blood pressure." 19

Sixty five of $117(56 \%)$ contained a section on smoking, and of these $57 \%(\mathrm{n}=37)$ mentioned monitoring (table). Fifty four per cent $(\mathrm{n}=35)$ provided no information or no specific (not useful in clinical practice) recommendations for what to monitor, $69 \%(n=45)$ for when to monitor, and $65 \%(n=42)$ for what to do if the target was out of range (table).

For smoking, the findings were less than ideal, as less than a third reported when to monitor, and often information was missing or was general — for example, "follow up should be incorporated as needed," 20 every opportunity," "every visit," 21 or "check smoking status annually." 22

Of the 41 guidelines reporting specific time intervals for lipid monitoring, $66 \%(\mathrm{n}=27)$ did not explicitly reference the evidence or the level of evidence used for these recommendations. Two of 14 reported a B level of evidence (controlled trials or no randomisation), and 12 of 14 were based on weak levels of evidence (non-analytical study or expert opinion). Seventeen of $28(61 \%)$ guidelines reporting specific time intervals for monitoring hypertension did not provide explicit reference for the associated evidence or the level of evidence. All 11 reporting a level of evidence were based on consensus or expert opinion, with eight based on one publication. ${ }^{23}$ Sixteen of 20 $(80 \%)$ guidelines reporting specific time intervals for monitoring of smoking cessation did not explicitly reference the evidence or the level of evidence for these recommendations.

\section{DISCUSSION}

More than half of the guidelines in our sample did not deal with the monitoring of one or more of the main risk factors for cardiovascular disease. Guidelines with a section on lipids or hypertension in which a specific paragraph on monitoring was present led to more frequent specific recommendations that would be useful in clinical practice. However, guidelines were often confusing and contradictory for monitoring recommendations.

It was rare to find a clear description of the monitoring phase in any guideline that would help clinicians to apply the recommendations clearly in clinical practice. In particular, the time intervals reported were often broad, with a wide margin of uncertainty. Time intervals for monitoring risk factors varied widely across the guidelines and even within the same guideline. Only a few guidelines offered different monitoring indications for those at low, intermediate, or high risk of cardiovascular disease.

Most of the guidelines did not explicitly reference evidence or the level of evidence underpinning the monitoring recommendations about "when to monitor." The vast majority that did reference evidence were based on consensus, expert opinion, or weak levels of evidence. This is likely to lead to uncertainty and confusion about when to monitor. Only a few guidelines declared that there was no evidence for the best strategy of monitoring. We believe this lack of evidence leads to wide variation in monitoring in clinical practice.

\section{Limitations of the study}

Our study has several limitations. Our main interest was on adequacy and completeness of information on monitoring so we did not carry out a formal quality evaluation of the guidelines by, for example, using the appraisal of guidelines research and evaluation (AGREE) instrument. The first reason was pragmatic: doctors rarely (if ever) apply such evaluation tools when using guidelines in their clinical practice. Furthermore, the checklist for appraisal of guidelines research and evaluation in particular considers the whole guideline and is not intended for individual recommendations. ${ }^{24}$ However, we did score guidelines based on whether they were the product of a national or international initiative, or developed by a guideline organisation or programme with high output, which has previously been shown to be a marker of high quality. ${ }^{25} \mathrm{~A}$ subgroup analysis of these guidelines 


\section{WHAT IS ALREADY KNOWN ON THIS TOPIC}

Monitoring regimens for cardiovascular disease (CVD) risk factors (lipid levels, hypertension, and smoking) could impact substantially on patients' outcomes, clinical decisions, and healthcare costs

Clinical guidelines aim to raise the overall quality of care by using best evidence to standardise the decision making process on diagnosis, management, and treatment

Most CVD management guidelines deal poorly with monitoring and no systematic examination has been done of monitoring recommendations in clinical practice guidelines

\section{WHAT THIS STUDY ADDS}

Only a fifth (23/117) of English language guidelines published or updated between 2002 and February 2009 dealt with monitoring of all three risk factors, and one or more risk factors was missing from more than half the guidelines

The monitoring recommendations in different guidelines were often confusing and contradictory

It was rare to find a clear description of the monitoring phase in any guideline that would help clinicians to apply the recommendations directly in clinical practice

showed similar results to the initial analysis, therefore failing to explain the problem by variability in quality between guidelines. We selected guidelines produced in Western countries because the effect of these risk factors is consistent in men and women, across different geographical regions, and by ethnic group. ${ }^{2} \mathrm{We}$ limited our eligibility criteria to guidelines published in English, but the broad sample we found meant that the results of the review covered mainly high income countries.

\section{Implications for practice and for the development of guidelines}

Improving the monitoring sections in guidelines on risk factors for cardiovascular disease could greatly improve the coordination of monitoring in clinical practice. Moreover, adequately stratifying the monitoring recommendation by individual risk should also substantially aid practice. Guideline developers should describe monitoring in a separate section for risk factors that require intervention and monitoring. When a monitoring process is described, this should include clear reporting of what to monitor, when, and what to do if something changes; along with clear referencing of the evidence sources and a comment on the strength of this evidence. If insufficient evidence is available to support a specific monitoring schedule, this lack of evidence should be declared explicitly, with a description of the new research that would fill the gap.

Contributors: IM, RP, and $\mathrm{CH}$ conceived the study and designed the protocol. IM and DB extracted the data. RP oversaw the statistical analysis. All authors had input into the writing of the manuscript and approved the final draft. $\mathrm{CH}$ is the guarantor of the data. Funding: This study was funded by a National Institute for Health Research Health Technology Assessment programme. Competing interests: All authors have completed the Unified Competing Interest form at www.icmje.org/coi_disclosure.pdf (available on request from the corresponding author) and declare: no support from any organisation for the submitted work; no financial relationships with any organisations that might have an interest in the submitted work in the previous three years, no other relationships or activities that could appea to have influenced the submitted work.

Ethical approval: Not required.

Data sharing: The full dataset is available from the corresponding autho at carl.heneghan@dphpc.ox.ac.uk.

1 Altman DG, Simera I, Hoey J, Moher D, Schutz K. EQUATOR: reporting guidelines for health research. Lancet 2008;371:1149-50.

2 Yusuf S, Hawken S, Ounpuu S, Dans T, Avezum A, Lanas F, et al. Effect of potentially modifiable risk factors associated with myocardial infarction in 52 countries (the INTERHEART study): casecontrol study. Lancet 2004:364:937-52.

3 Emberson JR, Whincup PH, Morris RW, Walker M. Re-assessing the contribution of serum total cholesterol, blood pressure and cigarette smoking to the aetiology of coronary heart disease: impact of regression dilution bias. Eur Heart J 2003;24:1719-26.

4 Ferket BS, Colkesen EB, Visser JJ, Spronk S, Kraaijenhagen RA, Steyerberg EW, et al. Systematic review of guidelines on cardiovascular risk assessment: which recommendations should clinicians follow for a cardiovascular health check? Arch Intern Med 2010;170:27-40.

5 Glasziou PP, Irwig L, Heritier S, Simes RJ, Tonkin A. Monitoring cholesterol levels: measurement error or true change? Ann Intern Med 2008;148:656-61.

6 US Department of Health and Human Services. Medicare national coverage determinations, manual coverage determinations. 2010. https://www.cms.gov/manuals/downloads/ncd103c1_Part3.pdf.

7 Glasziou P, Irwig L, Mant D. Monitoring in chronic disease: a rational approach. BMJ 2005;330:644-8.

8 European Stroke Organisation (ESO) Executive Committee; ESO Writing Committee. Guidelines for management of ischaemic stroke and transient ischaemic attack 2008. Cerebrovasc Dis 2008;25:457-507.

9 Graham I, Atar D, Borch-Johnsen K, Boysen G, Burell G, Cifkova R, et al. European guidelines on cardiovascular disease prevention in clinical practice: executive summary. Fourth Joint Task Force of the European Society of Cardiology and other societies on cardiovascular disease prevention in clinical practice (constituted by representatives of nine societies and by invited experts). Eur J Cardiovasc Prev Rehabil 2007;14(suppl 2):E1-40S

10 Canadian Stroke Network and the Heart and Stroke Foundation of Canada. Canadian best practice recommendations for stroke care. Canadian Stroke Network and the Heart and Stroke Foundation of Canada, 2006.

11 Campbell-Scherer DL, Campbell-Scherer DL, Green LA. ACC/AHA guideline update for the management of ST-segment elevation myocardial infarction. Am Fam Physician 2009;79:1080-6.

12 Fulcher GR, Amarena JV, Conner GW, Gilbert RE, Hankey GJ. Prevention of cardiovascular disease: an evidence-based clinical aid 2004. Med J Aust 2004;181(suppl 6):F4-14S.

13 NCEP Expert Panel. Third report of the National Cholesterol Education Program (NCEP) Expert Panel on detection, evaluation, and treatment of high blood cholesterol in adults (adult treatment panel III), final report. Circulation 2002;106:3143-421.

14 University of Michigan Health System. Management of type 2 diabetes mellitus: guidelines for clinical care. 2008. http://cme. med.umich.edu/pdf/guideline/dm08.pdf.

15 Goldstein LB, Adams R, Alberts MJ, Appel LI, Brass LM, Bushnell CD, et al. Primary prevention of ischemic stroke: a guideline from the American Heart Association/American Stroke Association Stroke Council: cosponsored by the Atherosclerotic Peripheral Vascular Disease Interdisciplinary Working Group; Cardiovascular Nursing Council; Clinical Cardiology Council; Nutrition, Physical Activity, and Metabolism Council; and the Quality of Care and Outcomes Research Interdisciplinary Working Group. Circulation 2006;113:e873-923.

16 JBS 2. Joint British Societies' guidelines on prevention of cardiovascular disease in clinical practice. Heart 2005;91(suppl 5):v1-52S.

17 National Vascular Disease Prevention Alliance. Consensus statement for the prevention of vascular disease. Aust Fam Physician 2004;33:235-9.

18 British Columbia Medical Association. Cardiovascular diseaseprimary prevention guidelines and protocols. 2008. www. bcguidelines.ca/gpac/pdf/cvd.pdf.

19 Dickstein K, Cohen-Solal A, Filippatos G, McMurray JJ, Ponikowski P, Poole-Wilson PA, et al. ESC guidelines for the diagnosis and treatment of acute and chronic heart failure 2008: the Task Force for the Diagnosis and Treatment of Acute and Chronic Heart Failure 2008 of the European Society of Cardiology. Developed in collaboration with the Heart Failure Association of the ESC (HFA) and endorsed by the European Society of Intensive Care Medicine (ESICM). Eur Heart J 2008;29:2388-442. 
20 Buse JB, Ginsberg HN, Bakris GL, Clark NG, Costa F, Eckel R, et al. Primary prevention of cardiovascular diseases in people with diabetes mellitus: a scientific statement from the American Heart Association and the American Diabetes Association. Circulation 2007;115:114-26.

21 Smith SC Jr, Allen J, Blair SN, Bonow RO, Brass LM, Fonarow GC, et al. AHA/ACC guidelines for secondary prevention for patients with coronary and other atherosclerotic vascular disease: 2006 update: endorsed by the National Heart, Lung, and Blood Institute. Circulation 2006;113:2363-72.

22 University of Michigan Health System. Secondary prevention of coronary artery disease. 2009. http://cme.med.umich.edu/pdf/ guideline/cad.pdf.
23 SIGN. Risk estimation and the prevention of cardiovascular disease: N 97. 2007. www.sign.ac.uk.

24 Chobanian AV, Bakris GL, Black HR, Cushman WC, Green LA, Izzo JL Jr, et al. Seventh report of the Joint National Committee on Prevention, Detection, Evaluation, and Treatment of High Blood Pressure. Hypertension 2003;42:1206-52.

25 Burgers JS, Cluzeau FA, Hanna SE, Hunt C, Grol R. Characteristics of high-quality guidelines: evaluation of 86 clinical guidelines developed in ten European countries and Canada. Int J Technol Assess Health Care 2003;19:148-57.

Accepted: 24 December 2010 